REVISTA ANDALUZA DE ANTROPOLOGÍA.

NÚMERO 11: TRABAJO Y CULTURAS DEL TRABAJO EN LA GLOBALIDAD HEGEMÓNICA

SEPTIEMBRE DE 2016

ISSN 174-6796

[pp. 121-145]

http://dx.doi.org/10.12795/RAA.2016.11.06

Recibido: 27/05/2016

Aceptado: 12/07/2016

\title{
ALMACENERAS. GÉNERO Y TRABAJO EN LOS ALMACENES DE MANIPULADO DE FRUTA EN LA REGIÓN DE MURCIA
}

\section{ALMACENERAS. GENDER AND WORK IN FRUIT PROCESSING PLANTS IN THE REGION OF MURCIA}

\author{
Elena Gadea Montesinos \\ Universidad de Murcia \\ Andrés Pedreño Cánovas \\ Universidad de Murcia \\ Carlos de Castro Pericacho \\ Universidad Autónoma de Madrid \\ Antonio J. Ramírez Melgarejo \\ Universidad de Murcia
}

\section{Resumen.}

La creciente importancia de las cadenas globales en la producción agrícola para fresco ha convertido los almacenes de manipulado de frutas y hortalizas en espacios centrales para las estrategias de acumulación de la agroindustria. Las innovaciones tecnológicas y organizativas asociadas a esta nueva centralidad se producen, sin embargo, en espacios de trabajo fuertemente segmentados y desvalorizados. Espacios que han tendido a movilizar fuerza de trabajo vulnerable, como aquella conformada por mujeres, generalmente en estrecha relación con la posición subordinada que éstas ocupan en las comunidades locales y los hogares en que viven. La feminización del empleo en los almacenes ha 
contribuido a conformarlo como una actividad estable pero discontinua, central pero precaria, postfordista pero minusvalorada socialmente. En la vida de las almaceneras, estas lógicas introducen una específica itinerancia entre el espacio productivo y reproductivo, una temporalidad cíclica y discontinua, que se traduce en un cultura y una identidad laboral particulares. El objetivo de este artículo es indagar en estas cuestiones a partir del estudio de la fruticultura de exportación de la Vega Alta del Segura, en la Región de Murcia. Para ello nos centraremos, de un lado, en el proceso de feminización del empleo en los almacenes de manipulado y, de otro, en las culturas e identidades laborales de las mujeres que trabajan en estos almacenes.

Palabras Clave: Culturas del trabajo, identidades laborales, género, segmentación laboral, agroindustria, territorio, localidades, hogares.

\section{Abstract.}

Packaging warehouses have become central points for accumulation strategies of agribusiness, due to the growing importance of global chains in fruits and vegetables production for fresh. Technological and organizational innovations associated with this process take place, however, in segmented and devalued workspaces. Packing warehouses have tended to use vulnerable work force, mainly women, in close relationship with the subordinate role they occupy in their households and communities. Because of the feminization, work in the packing warehouses is an activity stable but discontinuous, central but precarious, post-fordist but socially undervalued. In the life of women working in the packing stores, her job involves into a roaming between productive and reproductive space, a cyclical and discontinuous temporality, which brings about a culture and a particular work identity. In this paper, we analyze these issues from a case study: fruit growing export of Vega Alta del Segura, in the region of Murcia. To do this, we will focus on in the process of feminization of work in packing warehouses and in cultures and work identities of women in these workplaces.

Keywords: Labor cultures, labor identities, gender, labor segmentation, agribusiness, territory, local communities, household.

\section{INTRODUCCIÓN}

El almacén de manipulado o confección del producto agrícola ha adquirido una nueva centralidad productiva en la agricultura de frutas y hortalizas para mercados de fresco y exportación. Tal centralidad se deriva del papel hegemónico que el capital comercial tiene en la orientación de los productores agrícolas, de tal forma que el almacén integra ambos eslabones de la cadena, posibilitando los nuevos principios organizativos del 
salto postfordista dado por la producción de frutas y hortalizas: Just in Time, calidad y diferenciación del producto, cadenas de frío, imagen y marketing, etc.

En la agricultura intensiva de la Región de Murcia, como en muchos otros enclaves productivos agrícolas, el almacén es un espacio de trabajo intensivo en mano de obra altamente feminizada, lo que introduce una paradoja: siendo un espacio central de la cadena productiva, sus trabajadoras están en una especie de no-lugar, ni dentro ni fuera del todo. Su trabajo es central para la lógica postfordista de la nueva agricultura intensiva, pero su posición en la división del trabajo es tremendamente subalterna, lo que se traduce en una relación salarial y laboral precaria, mayoritariamente eventual, que en algunos casos adopta la forma de una estabilidad "fija-discontinua". La conformación de este empleo estable pero discontinuo, central pero precario, postfordista pero desvalorizado socialmente, subyace a las razones de fondo por las cuales se constituyó y se mantiene como propio de mujeres. Aunque en las últimas décadas las empresas han experimentado un profundo proceso de racionalización e innovación tecnológica, en la organización del trabajo han pervivido los motivos para seguir haciendo femeninas las tareas en los almacenes. En la vida de estas mujeres, la lógica del trabajo en el almacén introduce una específica itinerancia entre el espacio productivo y reproductivo, una temporalidad cíclica y discontinua, que se traduce en un cultura y una identidad laboral particulares.

El concepto de cultura de trabajo desarrollado por la antropología andaluza ofrece una perspectiva de indudable interés para los fines de este artículo. Partimos aquí de la definición de esta cultura como un "conjunto de conocimientos teórico-prácticos, comportamientos, percepciones, actitudes y valores que los individuos adquieren y construyen a partir de su inserción en los procesos de trabajo y/o de la interiorización de la ideología sobre el trabajo, todo lo cual modula su interacción social más allá de su práctica laboral concreta y orienta su especifica cosmovisión como miembros de un colectivo determinado" (Palenzuela, 1995: 13). Esta definición posibilita acercarse a las culturas del trabajo desde una perspectiva situada, interseccional, procesual, integradora de diversos ámbitos de la vida social y que permite vislumbrar las contradicciones y disputas sobre el significado dado al trabajo por los diferentes agentes.

Las culturas del trabajo se generan, como señala Moreno, en el marco de unas relaciones de producción determinadas que operan en contextos espacio-temporales definidos. Estas culturas, lejos de quedar contenidas en los espacios de trabajo, los desbordan, afectando "no sólo a lo relacionado con el ámbito laboral, a las prácticas, concepciones y valoraciones en relación a este, sino también a cuanto refiere a los demás ámbitos de la vida social y de las concepciones de los individuos" (Moreno, 1997: 20). Siguiendo a Reygadas (2002), al hablar de culturas del trabajo debemos estar atentos tanto a la "eficacia simbólica del trabajo" como a la "eficacia laboral de la cultura". En efecto, en el trabajo "se producen costumbres, signos de identidad, valores y tradiciones que 
desde ahí impregnan otras esferas de la vida social", de manera que los trabajadores y trabajadoras hacen uso, en los diversos espacios de su vida cotidiana, de normas, valores y representaciones que se crean y recrean en las relaciones laborales (Reygadas, 2002: 108). Por otro lado, "la cultura del trabajo no se genera de forma exclusiva en la actividad laboral, sino que tiene su origen en el conjunto de la producción simbólica de la sociedad", por lo que en la producción de significados sobre el trabajo "los agentes pueden recurrir a un capital simbólico más amplio, a la cosmovisión y a los valores propios de la cultura" (Reygadas, 2002: 112).

El hecho de que las culturas del trabajo tengan un carácter situado e integrador de diversos ámbitos de la vida social nos obliga, a la hora de aproximarnos a ellas, a ir más allá de los espacios de trabajo, a prestar atención a dos ámbitos de sociabilidad privilegiados: los hogares y las localidades. En relación al primero de estos ámbitos, es importante recordar que en la incorporación de los sujetos a la esfera productiva está involucrada su posición social en el ámbito reproductivo. No podemos, por tanto, separar producción y reproducción social. Para el tema que nos ocupa, no debemos obviar que la asignación de la mujer al ámbito doméstico se traduce en una posición desvalorizada en la esfera pública y, en particular, en el mundo laboral. Por otro, la incorporación al empleo, en términos de disponibilidad y aceptabilidad, debe situarse en el marco más general de las "estrategias de supervivencia" de los hogares y del contexto local como espacio de límites y posibilidades en el que distintas estrategias y "complejos agregados de reciprocidad y redistribución" pueden activarse (Mingione, 1993). Los contextos locales son fundamentales para comprender las culturas laborales, en particular en casos como el que estamos analizando, donde una especialización productiva, largamente gestada, convierte unos determinados oficios en signos de identidad local (Saglio, 1991).

A la consideración situada de las culturas del trabajo debemos sumar una perspectiva interseccional, atenta al modo en que operan distintos ejes de desigualdad. En este sentido, señala Moreno, el concepto de culturas del trabajo se inserta en una "matriz estructural" conformada por las relaciones de producción, las relaciones de sexo-género y las relaciones interétnicas, que generan "un sistema de identidades colectivas que son también estructurales: las identidades productivas (de clase y socioprofesionales), las identidades de sexo-género y las identidades étnicas" (Moreno, 1997:21). Inscribir las relaciones étnicas, de género y generación en el estudio de las culturas del trabajo es fundamental en el caso que estamos analizando, al tratarse de un espacio laboral tradicionalmente feminizado que, en las últimas décadas, ha experimentado una cierta diversificación en los perfiles de las trabajadoras, con la incorporación de jornaleras inmigrantes y de jóvenes con mayores niveles de cualificación.

Por último, las culturas del trabajo tienen un carácter dinámico y disputado. Como apunta Palenzuela, son realidades que "se construyen, cristalizan y modifican a 
través de un proceso histórico/temporal", de manera que "el estudio diacrónico de los comportamientos sociales, actitudes y valores de un colectivo nos mostrará las diferentes fases o adecuaciones por las que su cultura del trabajo ha transitado" (Palenzuela, 1995: 14). En estos procesos de construcción encontramos múltiples relaciones de poder, tanto dentro como fuera de los espacios de trabajo, por lo que las culturas del trabajo se convierten en espacios de disputa y en espacios disputados, donde se ponen en juego relaciones de dominación y de definición de los principios de visión y división del mundo social (Bourdieu, 1997).

El objetivo de este artículo es indagar en las culturas laborales de las mujeres que trabajan en los almacenes de manipulado de frutas en la agroindustria murciana. Para ello, en primer lugar, se realiza un abordaje procesual de la feminización del trabajo en los almacenes de manipulado agrícola, para tratar de detectar los cambios que se han producido en la configuración de este espacio laboral. La segunda parte del artículo se centra en el análisis de los significados que las mujeres otorgan al empleo en los almacenes y en sus representaciones en relación a las cualificaciones y temporalidades de su trabajo. La base empírica de estos análisis procede de una investigación ${ }^{1}$ realizada, entre 2012 y 2015, en un área de producción intensiva de fruta y uva de mesa en la Vega Alta del río Segura en la Región de Murcia comprendida por los municipios de Cieza, Fortuna, Abarán y Blanca. El enfoque metodológico empleado fue fundamentalmente cualitativo, a través de 60 entrevistas en profundidad a los diferentes actores sociales que conforman la red de posiciones del complejo frutícola (jornaleros del campo, trabajadoras de almacén, pequeños agricultores, cooperativas, grandes empresas agroexportadoras, etc.). Este trabajo de campo fue acompañado de inmersiones en el territorio de estudio, lo que posibilitó la recogida de numerosas observaciones etnográficas. Si como precisa Alba Rico, "lo que distingue un paisaje de un territorio es que, mientras que el paisaje es objeto de contemplación, el territorio es objeto de disputa" (Alba, 2011: 175), el diario de campo nos permitió acceder a ese territorio disputado donde palpita "el sabor y el dolor de la acción, el ruido y el furor de la sociedad que los pasos establecidos por las ciencias humanas ponen habitualmente en sordina, cuando no los suprimen completamente" (Wacquant, 2004: 15)².

\section{LOS ALMACENES DE MANIPULADO EN LA AGROINDUSTRIA MURCIANA: UN ESPACIO FEMINIZADO}

\subsection{El desarrollo de la agricultura industrial en la Región de Murcia}

1. La investigación se realizó en el marco del proyecto de I+D "Sostenibilidad social de los nuevos enclaves productivos agrícolas: España y México", dirigido por Andrés Pedreño Cánovas y financiado por el Ministerio de Ciencia e Innovación (2012-2014, CSO2011-28511).

2. Para una reflexión más amplia sobre los aspectos metodológicos de la investigación, véase Equipo Enclaves-Universidad de Murcia (2016). 
En la Vega Alta del Segura se desarrolló, desde finales del siglo XIX y las primeras décadas del siglo XX, un primer ciclo de agricultura comercial de vocación exportadora en torno a la producción frutícola, especializado en la producción de naranjas y de frutas de hueso destinadas a la conserva (Pérez Picazo y Lemenieur, 1994). Tras el freno que supuso la guerra civil española y la postguerra para el desarrollo de esta agricultura de exportación, en las décadas fordistas de los años 50 y 60 la demanda de los países europeos de alimentos no perecederos posibilita un segundo ciclo de expansión frutícola por el salto de la industria conservera a economías de escala, de tal forma que una nueva norma de producción vehicula el paso de una fase artesanal a una fase agroindustrial protagonizada por la gran fábrica y la centralización/concentración de capital (Martínez Carrión, 2002). En este largo ciclo frutícola destaca la población de Abarán donde se localiza en 1899 la primera fábrica de conservas de fruta ${ }^{3}$. A partir de entonces, los municipios de la Vega Alta del Segura experimentarán una identificación de la localidad con la cultura del trabajo de las conserveras y los almacenes de fruta ${ }^{4}$.

Tras la crisis de la conserva de las décadas de los años 70 y 80, debido a los cambios en las pautas de consumo alimentario a favor de los productos frescos y a las dificultades del sector para competir en unos mercados cada vez más internacionalizados ${ }^{5}$, se

3. Abarán acuña el lema "Abarán, París, Londres" para resaltar la vocación exportadora de sus producciones, una localidad que en los años 60 emerge como un emporio industrial proletarizado en torno a las fábricas conserveras. Valga, como ejemplo del juego de identificaciones entre el almacén frutícola, la localidad, su vida urbana y las mujeres abaraneras, esta canción popular recogida por el cronista José S. Carrasco Molina (2006) que data, según sus propias apreciaciones, de las décadas de oro de la conserva (años 50 y 60): A la entrada de Abarán / Lo primero que se ve / El almacén de Macanás / Y la casa del Chiqué / Más abajo está el Cervantes / Que es un gran Teatro / Con mucho valor / Tiene un lujazo tremendo / Y en el techo una araña / Que vale un millón / Viva Abarán, bonita población / Viva Abarán con su Calle Mayor / Viva Abarán y las chicas abaraneras / por donde quiera que vayan / les llaman las naranjeras.

4. La identificación entre localidad y culturas de trabajo ha sido ampliamente señalada por la antropología. Como señala Palenzuela, citando el trabajo de Saglio (1991), "cuando se produce una cierta concentración espacial de estos oficios, junto a una prolongada tradición productiva, se relacionan estas "culturas del trabajo" con la localidad que las acoge, convirtiéndose en un marcador de "identidad local" (Palenzuela, 1995: 18). Al igual que constata Téllez en su trabajo sobre el mantecado en Estepa, en nuestro territorio la fruticultura de exportación "ha llegado a convertirse en el motor económico de la localidad, rige el ciclo de vida de sus habitantes y es un referente de representación ideológica", por lo que "estamos ante una actividad industrial que al dotarse de elementos simbólicos, adquiere un carácter representativo para el colectivo (...), con ella se identifican incluso aquellos sectores de la comunidad no directamente implicados en el trabajo en las fábricas. (...) es mucho más que una simple actividad productiva, y se ha cargado de un contenido simbólico que la convierte en el principal referente de identificación local de este municipio en la actualidad" (Téllez, 2002: 212-213).

5. A finales de los 70 y principios de los 80 el sector conservero en la Región de Murcia afronta una importante crisis, con el cierre de muchas empresas en un contexto de reestructuración y concentración empresarial. Esta crisis fue resultado de la evolución negativa de las frutas y hortalizas en conserva "y de una cada vez menor rentabilidad empresarial debida a unos costes de producción y financieros crecientes” (Martínez, Martínez-Carrasco y Dios, 2005: 143). 
inicia un nuevo ciclo frutícola protagonizado por las frutas y uvas para mercados de fresco, ahondándose la vocación exportadora del territorio. Este tercer ciclo frutícola, que perdura hasta hoy, ha ido consolidando un número reducido de grandes empresas integradoras de todas las fases de la producción agroalimentaria, así como también grandes grupos cooperativos constituidos a partir de pequeños y medianos agricultores. En esta nueva dinámica de la globalización de las producciones, el almacén de manipulado y confección del producto agrícola adquiere una importancia decisiva y estratégica con el fin de responder, por un lado, a la demanda diversificada de los consumidores de los países desarrollados y, por otro, a las exigencias de adaptación a los criterios de las grandes estructuras de distribución, que ocupan una posición hegemónica en la cadena agroalimentaria. La fase de manipulación y confección del producto agrícola se ha convertido en un factor decisivo, no solo para atender a los nuevos mercados, sino también como principal estrategia de competitividad que permite a las empresas productoras mantener su posición en la cadena global agroindustrial. De hecho, lo que se produce en esta nueva lógica productiva es una mayor integración entre producción, transformación y comercialización, bajo una misma unidad técnico-económica.

A pesar de las importantes inversiones en tecnologías post-recolección que se han dado en la agroindustria murciana, el trabajo en el almacén de manipulado sigue teniendo elevadas exigencias de una mano de obra caracterizada por una norma de empleo de gran eventualidad, que se adapta perfectamente a los requerimientos específicos determinados por la transformación de los procesos productivos (persistencia de la estacionalidad y alta flexibilidad productiva y de tiempos de trabajo) y los modos de organización del trabajo, basados en métodos tayloristas y fordistas.

\section{2. la organización patriarcal del trabajo en los almacenes de manipulado}

La presencia de las mujeres en las tareas de transformación y manipulado del producto agrícola se remonta a los primeros desarrollos del ciclo frutícola en la Vega Alta del Segura. Una agroindustria que surge como un sector de pequeñas y medianas empresas de carácter familiar, con una regulación de tipo paternalista, bajo nivel de mecanización de los procesos productivos y un uso intensivo de la fuerza de trabajo. Un modelo de organización que configura un mercado de trabajo estacional y feminizado. Valga como esbozo de este primer ciclo la estampa que ofrece Castaño (2012) sobre la división del trabajo en los almacenes y las conserveras del Abarán de las primeras décadas del siglo $\mathrm{XX}$ :

"en la fábrica de conserva y fruta las condiciones eran similares en muchos aspectos, parte del trabajo se realizaba en la calle, la fábrica no tenía suficiente espacio y la vía no era transitable. Normalmente, los hombres realizaban sus labores en el campo y unos pocos en la fábrica; las mujeres que iban al campo solían ser 'mozas de servicio' que trabajaban por las tardes en plena faena de verano. Dentro de la 
fábrica ayudaban unos 6-8 hombres, los cuales se encargaban de lo más pesado y soldaban a mano la tapa de la conserva 'como un sombrero' con cobre y estaño. Sobre las mujeres habla de centenares, alrededor de 300-350. Fuera de la fábrica, en el campo, son en mayor número los hombres, aunque también trabajaban mujeres que macheaban la fruta" (Castaño, 2012: 352).

Varios elementos explican la feminización de este mercado de trabajo, en el que inicialmente se ocuparán mujeres locales procedentes de familias campesinas. En primer lugar, la fuerte interrelación de este sector con la comunidad local y las estrategias de supervivencia de los hogares. El empleo estacional y discontinuo de las mujeres en la conserva y en los almacenes responde a una estrategia de pluriactividad de la unidad familiar, como forma de captar rentas suplementarias.

La posición de estas mujeres en el mercado de trabajo agroalimentario se construyó, por tanto, como una continuidad de su posición en el orden agrícola tradicional, que vinculaba a la mujer a lo doméstico, con una presencia discontinua en el ámbito productivo, donde el trabajo se construye como ayuda (Narotzky, 1988). Una mano de obra construida socialmente en función de la categoría de género que definía en términos de "ayuda familiar" el valor de su fuerza de trabajo, con toda la estructura de percepciones y clasificaciones que ello implica, encajado en un relato hegemónico que considera que el lugar "natural" de la mujer es el hogar, y cuando trabaja lo hará puntualmente, para "ayudar" al cabeza familiar, y sin desatender las tareas que son "propias de su sexo", es decir, las relacionadas con el ámbito doméstico (Pedreño, Gadea y García, 2013). Una fuerza de trabajo con esas características resultó muy atractiva para las exigencias de flexibilidad y contención salarial de las empresas agrícolas, ya que la posición social de la mujer legitimaba la eventualidad y la precariedad.

Por último, la falta de alternativas laborales en estos territorios también jugará a favor de la feminización de la fuerza de trabajo. Esta zona se ha caracterizado, tradicionalmente, por una actividad agrícola insuficiente, que hace que los trabajadores tengan que poner en marcha estrategias de movilidad sectorial y territorial. Para las mujeres, responsables de la reproducción de los grupos domésticos, la movilidad no siempre era una opción y la cercanía de la actividad conservera y de manipulado compensaba las peores condiciones laborales, al permitirles compatibilizar el trabajo dentro y fuera del hogar. Esta estampa de recuerdos de la infancia de Gómez Sánchez (2002) es ilustrativa de la realidad que estamos describiendo:

“y los cientos de mujeres anónimas que con su trabajo hicieron y han hecho posible aún hoy día la economía de nuestro pueblo en el sector del manipulado de frutas? Eran verdaderas legiones de mujeres de nuestro pueblo junto con otras que venían de otros pueblos vecinos de Cieza y Blanca, las que en la época de la uva y el albaricoque trabajaban afanosamente para llevar un jornal a casa. Por cierto, ¿no os dice nada 
la palabra "velar"? Mujer abaranera trabajadora, en el almacén, en la fábrica, o en su casa, y que cuando llegaba el momento, también sabía ser señora y estar en su lugar" (Gómez Sánchez, 2002) ${ }^{6}$.

La itinerancia entre empleo y trabajo hogar de las mujeres se regula desde una construcción patriarcal y paternalista de la división social del trabajo. En el campo o en la conservera, la mujer hace trabajo por horas, estacional pero altamente flexible -en las épocas de mucha intensidad de fruta puede llegar a "velar", trabajando de noche-, pero al tiempo está al frente de su hogar como "señora". Su posición subalterna se desprende de esta regulación hogar-empleo. La organización familiar y comunitaria favorecía, por tanto, la constitución de un ejército de reserva femenino, altamente funcional a las necesidades de las empresas, y un mercado de trabajo segmentado sexualmente.

\subsection{De las fábricas de conservas a la producción para fresco en los almacenes: rupturas y continuidades}

A partir de los años 70, conforme la industria conservera y el manipulado se fueron modernizando, se inició un proceso de distanciamiento con la comunidad local, que también estaba en proceso de cambio. Los estrechos lazos que habían sustentado la explotación campesina y los primeros pasos de la industria conservera se resquebrajan. Las fábricas crecen considerablemente con la dinámica de producción de masa, los mercados de trabajo se ensanchan concurriendo masivamente trabajadores que vienen de localidades lejanas, los mecanismos reguladores institucionales introducen nuevas reglas de juego, aparece la conflictividad laboral y el sindicalismo como factor de regulación de las presiones competitivas de la economía hacia la comunidad local (Pedreño, 1998). Otro elemento que cuestionó el modelo de organización laboral de la conserva y los almacenes fue el creciente protagonismo de las nuevas mujeres trabajadoras, más jóvenes, con otro nivel educativo e incluso con una procedencia social extra agrícola. Estas mujeres ya no representaban su trabajo como ayuda familiar, y sus exigencias de reconocimiento desafiaban la división sexual del trabajo en el campo y en los almacenes (Pedreño, Gadea y de Castro, 2014). Como señala una representante sindical:

La mujer en aquellos años (...) que se inicia la actividad agroalimentaria y agrícola, entiende que la aportación de su trabajo a la economía familiar es de apoyo, no asume que ella es una parte más (...), teníamos que aceptar ese trabajo porque era el que había en el mercado laboral, pero eso no significaba que fuera menos digno socialmente, que no se nos respetara salarialmente o que no empezáramos a demandar los mismos derechos y el mismo tipo de relación laboral que el resto de trabajadores (E1_Elisa, representante sindical, autóctona, 60 años).

6. El término "velar" hace referencia a la práctica de trabajar de noche. Las mujeres regresaban al almacén después de cenar y trabajaban hasta las primeras horas de la madrugada. 
Con la expansión del sector servicios, muchas de estas mujeres abandonaron la actividad agrícola hacia ocupaciones más atractivas. Y a medida que las mujeres autóctonas abandonaban el empleo en la agroindustria, la presencia cada vez mayor de mujeres inmigrantes extracomunitarias posibilitó una nueva fuerza de trabajo a las empresas agrícolas. A principios de la década pasada, un informe del Consejo Económico y Social de la Región de Murcia (Rosas, Pérez y Segura, 2001) cuantificó la presencia de la mujer en los almacenes de manipulado: cerca del 75\% de los trabajadores eran mujeres y, el $60 \%$ de ellas, jóvenes de entre 21 y 40 años. Además la práctica totalidad eran mujeres de la propia comarca o de comarcas adyacentes. Desde entonces, a la feminización de las tareas de manipulación y de confección agroalimentaria se ha sumado una etnificación de la fuerza de trabajo. Las jornaleras extranjeras, con una alta presencia de mujeres procedentes de Marruecos y Ecuador, representaban a mediados de la década pasada el $74,2 \%$ de las trabajadoras de la agroindustria. Este proceso de etnificación ha sido menor en los municipios de la Vega Alta - donde la tradicional presencia de las mujeres en los almacenes se ha mantenido en gran medida- que en el conjunto de la Región de Murcia -especialmente en la zona de nuevos regadíos en la que la presencia de mujeres locales siempre ha sido menor?

A pesar de los cambios que se han producido en la organización del trabajo en los almacenes, este sigue siendo un espacio que mantiene las líneas tradicionales de segmentación sexual. La posición subordinada de las mujeres en los almacenes y fábricas de conserva se construyó y se sigue construyendo en una triple dimensión (Pedreño 1999).

En primer lugar, una fuerte diferenciación por género entre el trabajo manual y el trabajo de planificación, concepción, control y administración. La mayor parte de las mujeres están concentradas en la esfera del trabajo manual y prácticamente ausentes en la esfera del trabajo de concepción y control, aunque en los últimos años se observa un mayor número de encargadas mujeres. Esta línea divisoria marca, además, una diferenciación en la relación salarial. En la esfera del trabajo de planeación y control aparecen los contratos fijos, los salarios altos y en general una mayor estabilidad ocupacional, mientras que en la esfera del trabajo manual prevalece una plantilla contratada eventualmente, muy flexible en su relación contractual, el salario por horas y la inestabilidad.

En segundo lugar, desde el punto de vista de la división horizontal del trabajo, también se constata una clara división sexual del trabajo: las principales tareas realizadas por los varones son las de carga y descarga, transporte de las cajas y mantenimiento de la

7. En Abarán, Blanca y Cieza las mujeres autóctonas siguen manteniendo una presencia importante en los almacenes y en los campos, donde a mediados de la década pasada los contratos a jornaleras españolas representaban el 50\% del total de contratos a mujeres, frente al 25,8\% a nivel regional. Datos del Servicio de Empleo y Formación de la Comunidad Autónoma de la Región de Murcia. 
maquinaria; mientras que las mujeres se ocupan de la confección y envasado, selección y calibrado del producto. El trabajo se masculiniza cuando exige esfuerzo físico (cargar, descargar) o manejo de maquinaria y vehículos, y se feminiza cuando se caracteriza por ser más minucioso y habilidoso, capaz de seguir el ritmo marcado por las cintas transportadoras. De forma genérica puede afirmarse que la mujer ha sido excluida en los almacenes de manipulado del uso de herramientas y de máquinas, y concentrada en los trabajos más repetitivos e intensivos. Sin embargo, en los últimos años, en los almacenes de manipulado y confección de fruta y uva ha habido una importante mecanización de las tareas que tiene un efecto ambivalente: el esfuerzo físico es menor, pero las jornadas son muy largas y el ritmo de trabajo se ha intensificado. En definitiva, el cambio tecnológico ha supuesto para las mujeres trabajadoras de los almacenes una mayor vinculación de su tarea con las cualidades socialmente consideradas como femeninas (trabajo repetitivo, a ritmo, minucioso, etc.)

He estao desde julio a noviembre... Echando más horas que un reloj... Yo qué sé, el trabajo es muy cansao, pero ahora es mucho más cómodo que el de antes. O sea, yo trabajé hace pues 12 o 13 años (...) y antes, por ejemplo, era mucho más incómodo, o sea, estaba menos mecanizado, entonces tenías que hacer más esfuerzo físico. El de ahora, tú estás parada en tu sitio, eso sí es verdad, tú tienes un sitio aquí de tres metros, y ese es tu sitio. Estás como ahí, encajá, aunque no tengas barreras a los laos, tú estás ahí encajá y es: limpia uva, echa uva, limpia uva y echa uva. Le envasas y p’adelante (E4_Rosa, trabajadora de almacén, autóctona, 30 años).

Por último, construyendo el manipulado de frutas y hortalizas como un trabajo de y para mujeres, las empresas se garantizan el funcionamiento de las redes de reclutamiento de mano de obra a través de la proximidad, el parentesco o los vínculos de amistad. En un mercado de trabajo con escasas opciones ocupacionales para las mujeres, la estrategia empresarial de feminización del empleo es una forma de garantizar un flujo de trabajo estable y constante al tiempo que flexible y discontinuo.

\section{PRÁCTICAS Y VIVENCIAS DE LAS TRABAJADORAS DE LOS ALMACENES}

\subsection{Trabajar en los almacenes: sentidos en disputa}

Como veíamos en el apartado anterior, el empleo de las mujeres en los almacenes de manipulado y en las fábricas de conserva se había considerado tradicionalmente como una situación excepcional, debido tanto a su temporalidad como a la adscripción de la mujer al ámbito doméstico. Esta consideración del empleo femenino en la agroindustria se mantuvo mientras lo hicieron las condiciones sociales en las que se sustentaba y mientras el perfil de las trabajadoras fue homogéneo, tanto en el origen social de estas mujeres (mujeres locales de familias campesinas y obreras, hogares con presencia de varones ocupados en el rol de sustentadores principales) como en sus trayectorias 
laborales (entradas y salidas en el mercado laboral en función del ciclo vital de la familia). Al igual que Téllez en su trabajo sobre las mantecaeras en Estepa, encontramos en los almacenes de manipulado de la Vega Alta del Segura un trabajo con todas las características para no ser considerado como tal: una actividad temporal, intermitente, basada en cualidades más que en cualificaciones y adscrita al género femenino, lo que hace que las propias trabajadoras no consideren su trabajo "como una profesión, o que cuando lo hagan lo minusvaloren, o lo vean como una ayuda a sus grupos domésticos, o como algo siempre secundario a sus obligaciones de ama de casa” (Téllez, 2002: 193) . Esta prevalencia del papel reproductivo de las mujeres, por encima de su consideración como trabajadoras, sigue presente tanto en el discurso de una parte de las almaceneras, como en los relatos que sobre las mujeres de estas localidades se construyen desde otros ámbitos: las almaceneras son trabajadoras, pero ante todo son mujeres, con las cualidades y roles que los imaginarios y representaciones de género les atribuyen'. Con la incorporación a la agroindustria de mujeres con otros perfiles sociales, los sentidos del trabajo también se diversifican. Juegan aquí un papel fundamental las estrategias de supervivencia de los hogares ${ }^{10}$, la etnicidad y la generación.

Para las mujeres pioneras en la agroindustria ("las viejas", como las califican otras trabajadoras de almacén) y para otras mujeres que por la composición y organización de sus hogares ocupan una posición análoga ${ }^{11}$, el empleo en los almacenes sigue

8. A ello contribuye, además, que el trabajo sin contrato y sin cotización a la Seguridad Social haya sido una práctica muy extendida en el sector, que tradicionalmente ha presentado altos niveles de economía sumergida. Esto ha supuesto una exclusión de las prestaciones sociales por enfermedad, desempleo o jubilación. Las mujeres de los almacenes han estado, por tanto, excluidas de los derechos de ciudadanía que otorga el empleo, lo que contribuye a diluir su identidad como trabajadoras.

9. Sirva como ejemplo la siguiente cita de la conferencia "Abarán en sus mujeres", impartida por J. Carrasco Molina, cronista de esa población: "Mujeres cuya única solera ha sido el trabajo, cuyo único blasón ha sido el esfuerzo continuado en sacar adelante una casa, una familia, y al mismo tiempo demostrar su valía, su tesón y su arte poniendo su grano de arena en el desarrollo económico de Abarán, que sin ellas no habría sido posible, que no se hubiera producido sin tantas manos expertas en seleccionar y empaquetar nuestras selectas frutas para que pudieran competir más allá de la Garita, o en trabajar con tanto sacrificio esas lías de esparto que también dieron vida a nuestro pueblo en un pasado no tan lejano, o en tantos otras actividades en las que siempre las mujeres de Abarán, las de ahora y las que nos han precedido y han gastado su vida en condiciones mucho más penosas, se han distinguido. Pero todo ello armonizado y mezclado en un delicioso cóctel con un donaire y belleza difíciles de encontrar en otros lugares en la misma proporción" (el subrayado es nuestro).

10. Utilizamos aquí el concepto de estrategias de supervivencia de los hogares para referirnos a las formas en que los hogares se organizan, movilizando un conjunto de recursos, garantizar su supervivencia. Como señala Mingione (1993), los trabajadores y sus familias ponen en marcha diversas estrategias para garantizar su supervivencia, movilizando recursos que pueden proceder del trabajo de sus miembros o de fuentes externas, como el Estado, las organizaciones comunitarias o las redes sociales en que se integran.

11. Nos referimos a hogares en los que el sustentador (considerado) principal es un varón y las necesidades de cuidado son importantes, con lo que el trabajo de la mujer en el almacén sigue el modelo tradicional de "trabajo como ayuda". 
representándose hoy en día como una actividad laboral complementaria. Aunque cada vez ocupe más tiempo en sus vidas y su aportación sea más importante para la economía familiar, el empleo en los almacenes es para estas mujeres una ocupación secundaria en relación a su actividad en el ámbito doméstico. Se trata, principalmente, de mujeres autóctonas que viven en hogares donde hay más miembros ocupados (con lo que sus ingresos no se consideran determinantes para la economía familiar) y/o las necesidades de cuidado son importantes. En estos casos, el empleo de la mujer en el almacén es considerado desde el modelo tradicional de "trabajo como ayuda" (Narotzky, 1988). Es el caso de María, una mujer de 63 años que comenzó trabajando muy joven en los almacenes de manipulado y ha adaptado su incorporación y su dedicación a la actividad laboral a los distintos momentos del ciclo familiar y, en particular, a la crianza: abandono del empleo mientras los hijos son pequeños, incorporación con horarios reducidos cuando van al colegio, mayor dedicación conforme crecen y ganan en autonomía. Cuando le preguntamos por el mantenimiento de este modelo de incorporación al empleo en los almacenes responde lo siguiente:

Eso también sigue funcionando pero también hay muchísima gente nueva estudiante, muchísima. Gente con carrera y que no tiene trabajo, y en el verano entra muchísima estudiante en las vacaciones a echar dos o tres meses de trabajo. Hay mucha variedad de personas trabajando. Antes éramos amas de casa pero ahora mucha gente tiene estudios y como no tienen otra cosa pues se van allí. A parte de eso, las mamás pues se casan y tienen hijos y cuando están un poco grandecicos también se van (E18_ María, trabajadora de almacén, autóctona, 63 años).

Las almaceneras como María son consideradas, antes que trabajadoras, amas de casa y mamás, en oposición a las jóvenes estudiantes y gente con carrera. Junto a estos dos perfiles, diferentes en términos de edad y de formación, encontramos un tercero al que se contrapone el perfil tradicional de almacenera. La diversificación de las estructuras familiares $y$, en los últimos años, la crisis económica, han alterado este perfil, de manera que mujeres que iniciaron su trayectoria laboral siguiendo dicho modelo de complementariedad se han convertido en sustentadoras principales de sus hogares, lo que transforma la consideración que tienen de su empleo. Es el caso de Lola, que ronda los sesenta años y empezó a trabajar en el almacén cuando su marido se quedó en el paro y continua trabajando porque la pensión de su marido es muy baja y tiene que ayudar a sus hijos.

Me casé, he estao criando a mis hijos (...), entonces mi marido trabajaba y... Lo despidieron del trabajo. Y entonces ya, cuando a él lo despidieron, pues entonces yo me fui a trabajar. Empecé a trabajar ahí en esa cooperativa, y ya me quedé allí, y allí estoy (...), yo podría tener ahora mismo mis problemas medio resueltos, en cambio tengo tengo tres hijos, pero uno de ellos está en el paro tres años, y está viviendo a... 
a... a costa nuestra, y "mamá, la luz", y "mamá, el agua”, ¿y qué hacemos? (E7_ Lola, trabajadora de almacén, autóctona, 60 años).

Es también el caso de Tania, una mujer separada y con dos hijas que trabaja en un almacén de fruta desde mediados de los 90.

Hombre, la mujer que trabaja que es la ayuda de su marido, que es un sueldo de apoyo, pues sí que se toma sus vacaciones tranquilamente y se va a la playa y se lo pasa bomba. Pero las que somos las cabezas de nuestra casa, que dependemos de nuestro sueldo... yo no me lo puedo permitir. (...) Hay quien necesita más el estar ahí y tener su sueldo y otras lo pueden tener más relajado porque es como un apoyo a su casa, no es lo mismo, no es igual (...), no es lo mismo que las que somos las que llevamos las riendas de todo (E5_Tania, trabajadora de almacén, autóctona, 45 años).

Una situación similar es la que encontramos entre la mayoría de trabajadoras extranjeras. Como señalábamos en otro trabajo (Pedreño, Gadea y García, 2013), la incorporación de las jornaleras inmigrantes a la agroindustria murciana pone en cuestión la representación del trabajo de la mujer como ayuda, dada la centralidad que este tiene en el mantenimiento de sus hogares, tanto en origen como en destino. Los inmigrantes que se ocupan en la agroindustria murciana han configurado, por lo general, hogares de doble ingreso, donde tanto los varones como las mujeres se han incorporado al empleo. El proyecto migratorio y las mayores necesidades económicas de estos trabajadores (que en muchos casos han dejado una parte de la familia en el país de origen y deben enviar remesas para su mantenimiento), unidos a la vinculación legal entre residencia y empleo, favorecen la incorporación de la mujer al empleo como actividad principal. Esto no significa, por supuesto, que no encontremos entre las trabajadoras inmigrantes a mujeres que consideren su trabajo como una ayuda, ni que su posición frente al trabajo sea estática. El relato de Yaiza muestra la complejidad de las posiciones de estas mujeres.

Yo cuando llegué aquí, y hasta hoy, mi marido dice "no, tú no trabajas, tú métete a estudiar, haces lo que quieres y con los niños y aquí en casa y tal" (...).Ya llegué a una edad que dije "yo voy a trabajar" y él no quiso, pues salí yo y busqué un contrato, "ya el contrato lo tengo, mañana empiezo, si él no quiere... Y ahora estoy trabajando y estoy ayudando, pues pago las clases de mi hijo, compro cosas del niño, como él dice: "para un capricho", pero para mí no son capricho. (...) Ahora, por ejemplo, la gente que está en deuda porque han comprado casa y tal, hace falta que trabaje la mujer, porque si no esa familia no va a mantenerse (E20_Yaiza, trabajadora de almacén y delegada sindical, marroquí, 35 años).

Otro elemento que marca diferencias en las representaciones del empleo en los almacenes es la edad. Para las mujeres más jóvenes, la mayoría de ellas de procedencia social extra 
agrícola y con niveles formativos más altos, el empleo en el almacén tiene un sentido de excepcionalidad vinculado a la actual coyuntura económica. Buena parte de estas mujeres se incorporaron a las tareas de manipulado en un contexto marcado por el desempleo y la falta de oportunidades laborales. Para ellas, especialmente para las que tienen mayores niveles educativos y expectativas laborales, su empleo en los almacenes es considerada una actividad laboral provisional. Como otras jóvenes de la zona, Rosa y Violeta trabajaron en los almacenes en verano durante su etapa de estudiantes. Ahora, con treinta años y una trayectoria laboral breve y truncada por la crisis, han vuelto al almacén. Respecto a la situación que vivieron en su primera experiencia en los almacenes, destacan que ahora ha cambiado el perfil de las trabajadoras.

Violeta: Más mujeres, hay muchas más mujeres jóvenes. Hay muchísima gente que se ha salido de estudiar y se ha metido al almacén directamente a trabajar, porque no hay salidas en los estudios... No tienes trabajo, te cansas de estar estudiando pa no, pa luego no tener ná, entonces ya directamente se meten (...)

Rosa: Y mucha gente con carreras que está allí. (...) Ahora sí se nota mucho, antes no. Antes estaban las mujeres mayores y las que íbamos a echar el verano y punto pelota. (...) Ahora ahí te encuentras gente de otra clase, gente que no quiere estudiar, de 15 o 16 años; gente que ha estudiao, con carrera, y que no tiene, y gente que a lo mejor se ha salío de un módulo porque dicen, mira... ahí ven, que esto me lleva a ná (...). Y gente vieja por supuesto, o sea que hay de toas clases (E4_Violeta y Rosa, trabajadoras de almacén, autóctonas, 30 años).

La idea de que su empleo en los almacenes es una situación provisional planea durante toda la entrevista con estas jóvenes; de hecho, ellas se distancian de las mujeres que, como sus madres, han trabajado toda su vida en el almacén:

Violeta: La mía tiene 63 años y hasta que no tenga los 65... o 67, a ver cómo sale eso, ella no se retira. Pero es por lo que ya está acostumbrá, que es lo que están acostumbrás todas las mujeres de este pueblo.

Rosa: Están acostumbrás y son felices. (...) Son felices, están en el almacén. Yo me he pasao muy bien, pero ellas son felices de estar en el almacén. ¿Qué quieres que te $\operatorname{diga?}(\ldots)$

Violeta: Es lo que se han acostumbrao, es lo único que han tenío. (...) Y es la costumbre. Nosotras por ejemplo, yo por ejemplo a dónde... yo no me quiero quedar hasta que me retire en el almacén. Yo ojalá que mañana encuentre un trabajo de administrativo, que es lo que yo he estudiao. Y me voy fuera y... O sea, fuera, que me da igual lo que me salga, pero no quiero estar toa mi vida en el almacén. También tengo muy claro que una vez que empiece la campaña de este año de mayo, ojalá que me llamen de las primeras (E4_Violeta y Rosa, trabajadoras de almacén, autóctonas, 30 años). 
La última parte de este fragmento muestra la ambivalencia con la que estas jóvenes viven su empleo en los almacenes. De un lado, es una actividad que no quieren realizar durante más tiempo del necesario; de otro, piensan que es la única opción laboral que tienen en estos momentos y necesitan ese empleo para obtener algún dinero que les permita cierta autonomía. Pero el rechazo al almacén va más allá de la actividad que realizan. Para estas jóvenes, el almacén no representa solo un ámbito de trabajo, sino un modo de vida y una representación a escala del espacio local. Los almacenes, sobre todo aquéllos de mayor antigüedad y tamaño, constituyen lugares centrales en la vida de los municipios que estamos analizando. No sólo organizan los tiempos y tareas durante las campañas sino que, a modo de plaza pública, son el espacio en el que se transmiten las informaciones, corren los rumores, se reproducen jerarquías, se ejerce el control social, se escenifican conflictos y las trabajadoras se enemistan y se enamoran.

Rosa: Tú no te puedes basar sólo en el almacén, porque como te metas en eso te conviertes en almacenera, si es lo que pasa, que te conviertes en almacenera. (...) Pero te lo tienes que tomar con la filosofía de 'vengo a trabajar, paso de to lo que hay alrededor y me voy', porque si no te amargas viva, eso sí es verdad. Si tú te ves ahí 12 horas pará, te amargas viva.

Violeta: Y luego las compañeras... Si le prestas mucha atención a las mismas compañeras, sales amargá del almacén, porque son almaceneras... de toa la vida, y son... son malas (...).

Rosa: Yo prefiero a dos gitanos malos que una almacenera mala (...), es verdad, es otra cultura. Igual que los gitanos son otra cultura, pues los almaceneros son otra cultura. Y las almaceneras malas son malas, pero malas por naturaleza. (...) De hacerte la vida imposible si pueden, ¿eh? (E4_Violeta y Rosa, trabajadoras de almacén, autóctonas, 30 años).

Resulta reveladora, del tipo de relaciones que se generan en el almacén y de la consideración social de sus trabajadoras, la imagen que estas jóvenes, que tratan de distanciarse de ese mundo laboral, tienen de lo que ellas definen como "almaceneras", una imagen que remite en última instancia a una dimensión moral. La categoría de almacenera no se aplica, como muestran los discursos, a cualquier trabajadora de almacén. Las jóvenes con estudios a la espera de un empleo mejor y acorde con sus cualificaciones no son almaceneras, tampoco lo son las trabajadoras inmigrantes. La figura prototípica de la almacenera es una mujer de la localidad, con una trayectoria laboral en el almacén larga y discontinua, pero definida principalmente como madre y esposa.

El término almacenera remite, por tanto, a esa construcción originaria de las mujeres en los almacenes que hoy ha adquirido una connotación negativa: las almaceneras son mujeres mayores, simples, cotillas y envidiosas, que no tienen otra cosa ni aspiran a 
otra cosa. Estos discursos contrastan con los relatos que, como hemos vistos, enaltecen la capacidad de esfuerzo y sacrificio de las mujeres abaraneras y ciezanas. Sin embargo, ambas imágenes contribuyen a diluir la identidad laboral de las mujeres que trabajan en los almacenes y a convertir su trabajo en un espacio clausurado para el reconocimiento.

\subsection{Tareas descualificadas, habilidades tácitas y disposiciones}

Las cualificaciones y destrezas que caracterizan el trabajo de las mujeres en los almacenes es un elemento de la cultura laboral fuertemente impregnado de las representaciones de género. La feminización de este espacio laboral y su reciente etnificación han permitido mantener "la secular construcción social del trabajo agrícola como trabajo desvalorizado, es decir, trabajo simple y eventual" (Pedreño, 1999:183). Aunque los almacenes se han convertido en espacios centrales para las nuevas lógicas de acumulación de la agroindustria, y aunque encontramos en ellos nuevas formas de organización del trabajo vinculadas a los protocolos de calidad y a las exigencias de la distribución que cambian las cualificaciones necesarias, esto no se ha producido en un reconocimiento a nivel de categorías laborales. El trabajo de las almaceneras sigue siendo considerado, como señala una representante sindical, un trabajo descualificado:

no hemos conseguidosalirdela categoría deauxiliar, deencajadora, empaquetadora... no cualificada. (...) Hoy en cualquier centro de manipulación las mujeres están acostumbradas a utilizar un ordenador porque es el que dice que se inicie un proceso. Mujeres que no tienen en su categoría especialización alguna son las que lo programan para decir "vamos a trabajar con un formato de tantos gramos, que fue recolectado tal día y que ha sido manipulado hoy", para que cuando sea comprado la persona sepa (E1_Elisa, representante sindical, autóctona, 60 años).

Con todo, como señalábamos en un trabajo anterior, la presencia permanente de las mujeres en los almacenes se ha traducido en una mayor apertura en el acceso a determinadas tareas y funciones que estaban vedados según la estructura tradicional de diferenciación laboral según género. Esto se constata en la mayor presencia de mujeres en funciones y tareas más cualificadas y mejor reconocidas, tradicionalmente masculinizadas, como encargadas o responsables administrativas (Pedreño, Gadea y García, 2013). A pesar de estos cambios, la desvalorización del trabajo de las mujeres en la agroindustria sigue funcionando y se entrelaza con las representaciones de género, de manera que la profesión de almacenera participa de la naturalización de saberes y competencias laborales que muchas investigaciones han señalado para el trabajo femenino (Narotzky, 1988; Maruani, 1991; Kergoat, 1994). Se produce así una retroalimentación entre tareas y cualificaciones: actividades repetitivas para las que no se requieren cualificaciones específicas sino, más bien, determinadas disposiciones. La tarea tediosa y monótona es ideal para la mano de obra considerada paciente y resistente (Mingo, 2013). La capacidad de aguante frente a largas jornadas, tareas repetitivas y posturas dolorosas, se convierte en el aval laboral por excelencia de las almaceneras. 
Yo entré a trabajar un miércoles por la tarde y miércoles por la tarde, jueves, y sábado a mí me dolían las pestañas. Tú imagínate estar así [encorvada] doce horas. (...) Yo tenía, a la hora, aquí [en las cervicales] un dolor que me moría. (...) Las bolsas las haces aqui y es: coge bolsa, hazla, coge bolsa, hazla, como no la tienes que pesar, es así todo el tiempo. Entonces esto de aquí [las cervicales] se te carga mucho. Yo por ejemplo, claro, yo llegué allí con una contractura, o sea, yo venía del fisio cuando me fui a trabajar, que me quité las pegatinas ésas pa irme a trabajar (E4. Rosa, trabajadora de almacén, autóctona, 30 años).

Las mujeres entrevistadas hablan de su capacidad de aguante como una condición sine qua non del trabajo en el almacén, pero no deja de haber en sus relatos un cierto tono de orgullo por su resistencia frente a un trabajo duro que deja huella en sus cuerpos. Contracturas en el cuello y en la espalda como consecuencia de posturas forzadas mantenidas durante horas en las cintas de envasado, varices en las piernas debido a las largas jornadas en pie, dolencias que se convierten en seña de identidad de las almaceneras y que se combaten mediante cualquier remedio que les permita seguir aguantando.

¡Anda, si yo te dijera a ti! i¿Nolotil?! De... que tengo también, el riñón tocao de... Neurofren. (...) Yo me tomao... Yo, y mis compañeras, hemos vivio a base de Neurofren, porque es la única manera de... Además, yo me fui a la baja, no me fui a la baja por el dolor de brazo, cuidao, me fui a la baja de tanto Neurofren que tomaba. Y pa poder dormir, relajantes, que llegó un momento que la boca, no podía ni hablar, y me quedaba, estaba como adormilá, de tanta cosa como tomaba. Entonces me creía que me había dao algún derrame cerebral o algo, y por eso fui a la doctora (...) yo me he ido del trabajo rabiando a que me... a que me pincharan, he vuelto a trabajar, burras, burras. De eso no tiene nadie la culpa, na más que yo. O la necesidad.... O la necesidad (E7_Lola, trabajadora de almacén, autóctona, 60 años).

Esta capacidad de aguante se forja en unas trayectorias laborales y en unas vivencias cotidianas que se transmiten en el seno de los hogares. En los municipios de la Vega Alta del Segura, el empleo en las fábricas de conserva o los almacenes de manipulado está indisolublemente unido a la imagen de las mujeres locales. La desvalorización de su trabajo en el sistema productivo y su posición subalterna en las relaciones de género al interior de los hogares son las dos caras de la misma moneda: la invisibilización del rol central que ejercen las mujeres como sostenedoras de la economía local y de la economía doméstica. Ante esta situación construyen su identidad, generación tras generación, como mujeres fuertes con capacidad de resistencia y aguante, capaces de administrar tiempos y recursos, ejes vertebradores de la vida social y económica que tratan de no ceder al desaliento o a la enfermedad. 
Las almaceneras más mayores comenzaron a trabajar cuando eran muy jóvenes, la mayoría por la necesidad de contribuir a una economía familiar siempre precaria.

¿Cuándo empecé yo? Pues tenía diez años... Hace ya.... Tengo 57... Todos esos. Cuando yo empecé a trabajar en el almacén tenía diez años, ese año cumplía los once (...) Entonces empezábamos todas bastante jovencitas, porque normalmente siempre había problemas en la casa también. (...) Y la mayoría de zagalas de diez, doce años, catorce... pero de diez y doce había mucha gente (E18_María, trabajadora de almacén, autóctona, 63 años).

La vinculación entre la localidad y el empleo en los almacenes, mantenida a lo largo de más de un siglo, hace que la mayoría de la población tenga experiencia directa de la dureza del trabajo y de la capacidad de aguante de las almaceneras. Lo han visto en sus abuelas, sus madres, sus hermanas... se ha encarnado como un elemento fundamental de la identidad de las mujeres en estos territorios.

Yo, por lo menos, mi madre con tres hijos yo no sé cómo lo ha hecho, porque además antes se echaba quince horas en el almacén. (...) Entonces yo me acuerdo de venir mi madre, o sea, cuando yo era un poquico así más mayorcica, de venir mi madre de trabajar por la noche a lo mejor a las nueve de la noche, de hacernos la cena o de vernos pa acostarnos, $y$ después irse otra vez a trabajar a velar, porque antes se estilaba lo de velar. Velar es hasta las dos de la mañana, hasta las tres de la mañana, para volver al día siguiente a las siete. (...) Y asi un día y otro (E4_Rosa, trabajadora de almacén, autóctona, 30 años).

Junto a esto, la incorporación de las jóvenes al empleo de manipulado durante el verano en su etapa estudiantil constituía, y todavía constituye, una especie de rito de paso que inscribe el almacén en las trayectorias laborales vitales de muchas jóvenes del municipio.

\subsection{Los tiempos del trabajo en el almacén: excepcionalidad y autoexplotación}

Los tiempos del trabajo constituyen un aspecto fundamental de las identidades y culturas laborales (Téllez, 2002; de Castro, 2012). Su duración a lo largo del año, sus horarios, sus ritmos, resultan indispensables para conocer cómo los trabajadores y trabajadoras viven e interpretan sus experiencias laborales. Como ya hemos señalado, el empleo en los almacenes de la Vega Alta del Segura se caracteriza por su estacionalidad, lo que marca una temporalidad que supedita los periodos de trabajo a las diferentes campañas de recogida de la fruta. Este carácter estacional del empleo en los almacenes marca una discontinuidad de la actividad laboral a lo largo del año que se ha incorporado de diversas formas a la gestión de la mano de obra y que marca relaciones contractuales diferentes. Encontramos, de un lado, a trabajadoras fijas-discontinuas, que mantienen con las empresas agrícolas una relación laboral estable (son fijas) pero limitada a determinados periodos (son discontinuas). De otro lado, los empresarios agrícolas hacen frente a las 
mayores necesidades de mano de obra recurriendo a la contratación temporal, bien directamente bien a través de trabajadores cedidos por empresas de trabajo temporal, una modalidad que ha aumentado en los últimos años.

En uno y otro caso, la discontinuidad de la actividad laboral, y la falta de alternativas en otros sectores, hace del empleo en estos territorios un bien escaso. La incertidumbre sobre la obtención y la duración de la actividad laboral favorece en las trabajadoras de los almacenes una actitud frente al trabajo que podríamos resumir como "echar horas". Una disposición a la autoexplotación altamente funcional al modelo agroindustrial, por su papel en la disponibilidad de fuerza de trabajo y en el disciplinamiento de las demandas laborales.

La empresa dijo que si queríamos [cobrar] las horas extraordinarias, entonces ellos se acogían a su derecho a que echáramos na más que ocho, entonces ya tropezamos con el problema de que queremos ganar to el dinero posible (...), cuando entró la UGT, se habló de que las pagaran extraordinarias, entonces claro, el jefe dijo, "bueno, pues yo, echáis ocho horas y así no os pago horas extraordinarias. Echáis, aunque tenga que venir más gente", claro, entonces las mujeres dijeron "Ocho horas, jmadre mía!, si trabajamos dos meses al año, ¡cómo vamos a trabajar na más que ocho horas, pos lo que queremos es echar veintisiete!", ¿me entiendes? (...). Si no tenemos otra cosa, si no tenemos otra cosa (E7_Lola, trabajadora de almacén, autóctona, 60 años).

Los horarios dependen del momento de la temporada y de los tiempos de la distribución. Es una organización de los tiempos adaptada completamente a las lógicas Just in Time que estructuran la cadena agroalimentaria.

El final estamos mucho tiempo echando pocas horas pero al principio empiezas a lo mejor un día que vas cuatro o cinco horas pero a lo mejor a los dos o tres días empiezas ya a echar diez (...) En plena temporada pues once horas, once y media es lo que echamos tres meses... Vamos a las siete y salimos a las ocho... (E18_María, trabajadora de almacén, autóctona, 63 años).

Esta gestión Just in Time de los horarios, supeditada a las demandas del mercado, se traduce para las mujeres de los almacenes en una imposibilidad de organizar el tiempo y las obliga a una disponibilidad permanente de su fuerza de trabajo (Pedreño, 1999).

En la pizarra nos lo ponen [el horario de trabajo] de una día para otro. Cuando tú sales... si no va a haber trabajo para mañana y tú tienes que venir a las 3 de la tarde pues te ponen "hasta tal número por las mañanas y de tal número para abajo... a las 3". Entonces tú si un día te llaman a las 3 de la tarde en vez de salir a las 18.30 como normalmente saldrías en tu turno pues sales a las 20.15... y ya si hay más trabajo piden voluntarias, puedes completar tus ocho horas hasta que haya trabajo (E5_Tania, trabajadora de almacén, autóctona, 45 años). 
En un contexto donde el empleo viene marcado por la escasez y la incertidumbre, la hiperflexibilidad y la disponibilidad constituyen elementos fundamentales de la cultura laboral de las almaceneras. La temporada de la fruta es, para estas mujeres, un tiempo dedicado exclusivamente al trabajo, donde toda actividad que no remita a la mera reposición de la fuerza de trabajo queda en suspenso. Esto introduce una tensión entre los requerimientos del almacén y los del ámbito doméstico, del que estas mujeres son en última instancia responsables. Para minimizar esta tensión, la tradicional feminización del trabajo de manipulado ha cristalizado, en algunos almacenes, en una organización de los tiempos de trabajo que trata de facilitar que las mujeres puedan encargarse del trabajo reproductivo. Por ejemplo, la jornada partida con tres horas de descanso a mediodía les permite recoger a sus hijos del colegio y estar en sus casas para organizar la comida. Lo mismo sucede con los pluses por asistencia al trabajo -como forma de evitar el absentismo sin penalizarlo excesivamente- y con la flexibilidad para incorporarse o abandonar la temporada de trabajo. Podríamos apuntar que, sabedoras de que la disponibilidad y vulnerabilidad de la fuerza de trabajo femenina se origina en su posición en el hogar, las empresas han favorecido el mantenimiento de ese perfil de trabajadoras, garantizándose así una mano de obra con esas características.

\section{CONCLUSIONES: SOBRE LAS CULTURAS DEL TRABAJO DE LAS MUJERES ALMACENERAS}

En la agroindustria murciana las mujeres han sido, tradicionalmente, la mano de obra mayoritaria en las fábricas de conserva y en los almacenes de frutas. El empleo de estas mujeres presenta un carácter paradógico ya que, siendo central para la lógica postfordista de la nueva agricultura intensiva, ocupa una posición subalterna en la división del trabajo, precaria en sus condiciones laborales y salariales, y sometida a la incertidumbre y la temporalidad. En la vida de estas mujeres, el almacén introduce una específica itinerancia entre el espacio productivo y reproductivo, una temporalidad cíclica y discontinua, que se traduce en un cultura y una identidad laboral particulares.

Desde sus inicios en las primeras décadas del siglo XIX, el empleo de las mujeres en los almacenes y las conserveras se desarrolló en íntima conexión con las estrategias de los hogares y con la imagen de la comunidad local: un trabajo considerado como ayuda que, de manera excepcional, sacaba a las mujeres del hogar para llevarlas a los almacenes y que llegó a convertirse en signo de identidad local. En las últimas décadas, se han producido importantes transformaciones en el ámbito productivo y en la sociedad en su conjunto que han puesto en cuestión algunos de los principios que sostenían la segmentación sexual del trabajo en los almacenes. Cabe señalar aquí diversos procesos, tanto endógenos como exógenos. Entre los primeros destacarían los cambios en la composición social de la fuerza de trabajo femenina, por la progresiva entrada de mujeres jóvenes e inmigrantes, que posibilitaron presiones y conflictos por la destradicionalización de las relaciones de 
trabajo. Entre los segundos, es indudable que la incorporación de las mujeres al mercado laboral ha venido modificando la sensibilidad y las percepciones sociales sobre su contribución en la división social del trabajo tanto en la esfera mercantil (reconocimiento del valor aportado) como en la esfera doméstica (preocupación por la conciliación, la cuestión de los cuidados, etc.).

A pesar de estos cambios, podemos destacar varios elementos de continuidad en la construcción generizada del trabajo en el almacén, tanto en la organización laboral como en la cultura laboral de las almaceneras. En primer lugar, en los significados que las mujeres dan a su empleo sigue muy presente la subordinación su rol laboral a las responsabilidades domésticas, a pesar de la mayor diversidad que introduce la presencia de jornaleras inmigrantes y de jóvenes con mayores niveles de cualificación. De hecho, el concepto de almacenera no se aplica por igual a todas las trabajadoras, sino que se reserva para las mujeres de la localidad, con una trayectoria de trabajo en el almacén larga y discontinua, pero definidas principalmente como madres y esposas. El término almacenera remite, como hemos visto, a esa construcción originaria de las mujeres en los almacenes que hoy ha adquirido una connotación negativa que contrasta con las imágenes que enaltecen la capacidad de esfuerzo y sacrificio de las mujeres locales. Sin embargo, en ambos casos se trata de representaciones que contribuyen a desdibujar su identidad laboral y a convertir su trabajo en un espacio clausurado para el reconocimiento. En segundo lugar, las tareas que las mujeres desempeñan en el manipulado siguen categorizándose como descualificadas, más relacionadas con determinadas cualidades consideradas como femeninas que con cualificaciones profesionales. Cualidades que, como en la definición de las almaceneras, remiten al campo semántico de las virtudes religioso-morales, aunque ahora con una carga positiva: obediencia, paciencia, esfuerzo, resistencia, aguante. Cualidades que se encarnan, de generación en generación, en estas mujeres de Abarán o de Cieza que han visto desde pequeñas cómo sus madres, sus tías y sus hermanas trabajaban largas horas en el almacén sin dejar de ser, nunca y ante todo, las señoras de sus casas.

En tercer lugar, la escasez y la incertidumbre que caracterizan el empleo en los almacenes hacen quelahiperflexibilidad yla disponibilidad se conviertan en elementosfundamentales de la cultura laboral de las almaceneras. Encontramos en estas mujeres una disposición a la autoexplotación que resulta muy rentable para el modelo agroindustrial, que puede hacer uso de una fuerza de trabajo abundante, disponible y disciplinada. La temporada de la fruta constituye, para estas mujeres, un tiempo dedicado exclusivamente al trabajo, donde toda actividad que no remita a la mera reposición de la fuerza de trabajo, todo aquello que impida a estas mujeres "echar" todas las horas posible, queda en suspenso.

El análisis de la organización y las culturas del trabajo de las mujeres en los almacenes y conserveras de la agroindustria murciana nos muestra unos espacios donde el género se 
ha puesto al servicio de las estrategias empresariales de competitividad y de configuración de un ejército de reserva capaz de responder a los ritmos de la distribución. El sentido que las mujeres otorgan a su trabajo, su consideración como descualificado y la vivencia de la temporalidad convierte a estas mujeres en una mano de obra altamente funcional a las exigencias de disponibilidad, flexibilidad y contención salarial de la agroindustria. A pesar de los cambios que se han producido, en las relaciones de trabajo del modelo agroexportador siguen perviviendo importantes contradicciones de clase que se anudan profundamente con las desigualdades de género, etnia y generación para mantener una norma de competitividad que se sostiene sobre la vulnerabilidad del trabajo y los bajos salarios. 


\section{REFERENCIAS BIBLIOGRÁFICAS}

Alba Rico, Santiago (2011) "De Paisaje a territorio: tres días en el sur de Túnez (I). Gafsa”. En José Daniel Fierro y Alma Allende (Santiago Alba Rico) Túnez, la revolución. Hondarribia: Sedicciones 28 - Editorial Hiru.

Bourdieu, Pierre (1997) Razones prácticas. Sobre la teoría de la acción. Barcelona: Anagrama.

Castaño Molina, Ma Ángeles (2012) "Mujeres Trabajadoras: las abaraneras de los años cuarenta”. Actas II Jornadas de Investigación y Divulgación sobre Abarán y el Valle de Ricote, 20 y 27 abril, pp. 341-355.

Castro, Carlos de (2012) "Algunas historias de los trabajadores. Las experiencias temporales y las identidades narrativas de los trabajadores". Revista Internacional de Sociología 70 (2), pp. 423-444.

Equipo Enclaves-Universidad de Murcia (2016) “¿Cómo hicimos el proyecto de investigación enclaves: Sostenibilidad social de los enclaves de agricultura intensiva: España y México" (2012-2015)??. Sociología del Trabajo 86, pp.107-124

Gómez Sánchez, A. (2002) "Estampas de una infancia”. En V Curso: Abarán, acercamiento a una realidad. Abarán: Centro de Estudios Abaraneros.

Kergoat, Danièle (1994) "Por una sociología de las relaciones sociales. Del análisis crítico de las categorías dominantes a una nueva conceptualización". En Cristina Borderías, Cristina Carrasco y Carmen Alemany (coords.) Las mujeres y el trabajo. Rupturas Conceptuales. Madrid: FUHEM-Icaria, pp. 515-527.

Martínez Carrión, José Miguel (2002) Economía de la Región de Murcia. Murcia: Editora Regional de Murcia.

Martínez Paz, José Miguel; Martínez-Carrasco Pleite, Federico y Rafaela Dios Palomares (2005) "La industria de conservas vegetales de la Región de Murcia: análisis de eficiencia técnica”. Revista de estudios regionales, nº 73, pp. 141-158.

Maruani, Margaret (1991) "La construcción social de las diferencias de sexos en el mercado de trabajo". Revista de Economía y Sociología del Trabajo 13-14, pp. 129-137.

Mingione, Enzo (1993) Las sociedades fragmentadas. Una sociología de la vida económica más allá del paradigma del mercado. Madrid: Ministerio de Trabajo y Seguridad Social. Mingo, Elena (2013) "Género y relaciones laborales en la agroindustria frutícola del Valle de Uco, provincia de Mendoza, Argentina”. Revista Iluminuras, 14 (33), pp. 244-261.

Moreno Navarro, Isidoro (1997) "Trabajo, ideologías sobre el trabajo y culturas del trabajo". Trabajo. Revista Andaluza de Relaciones Laborales 3, pp. 9-28. 
Narotzky, Susana (1988) Trabajar en familia. Mujeres, hogares y talleres. Madrid: Consejo Superior de Investigaciones Científicas

Palenzuela, Pablo (1995): "Las culturas del trabajo. Una aproximación antropológica", Sociología del Trabajo 24, pp. 3-28.

Pedreño, A., Gadea, E. y de Castro, C. (2014) "Labor, Gender and Political Conflicts in the Global Agri-food System. The case of Agri-export model of Murcia, Spain". En Alessandro Bonanno y Josefa Salete Cavalcanti (eds.) Labor Relations in a Globalized Food. Bingley: Emerald, pp. 193-214.

Pedreño, A., Gadea, E. y García, A. (2013) "Jornaleras de la globalización en el campo murciano", en Martha Judith Sánchez e Inmaculada Serra (coords.) Ellas se van, Mujeres migrantes en Estados Unidos y España. México: Instituto de Investigaciones Sociales UNAM.

Pedreño, Andrés (1998) "Economía flexible y ruralidad: el caso de la Vega Media del Río Segura en la Región de Murcia”. En Josep Ybarra Economía sumergida: el estado de la cuestión en España. Murcia: UGT.

Pedreño, Andrés (1999) “Taylor y Ford en los campos. Trabajo, género y etnia en el cambio tecnológico y organizacional de la agricultura industrial murciana". Sociología del Trabajo, 35, pp. 25-56.

Pérez Picazo, Ma Teresa y Lemeunier, Guy (1994) "La evolución de los Regadíos Mediterráneos. El caso de Murcia (ss. XVI-XIX)”. En VV.AA. Agriculturas mediterráneas y mundo campesino: cambios históricos y retos actuales. Almería: Instituto de Estudios Almerienses - Diputación de Almería.

Reygadas, Luis (2002) "Producción simbólica y producción material: metáforas y conceptos en torno a la cultura del trabajo". Nueva Antropología, XVIII (60), pp.101-119. Rosas, P., Pérez, M. y Segura, P. (2001) Condiciones de trabajo en el sector agroalimentario de la Región de Murcia. Murcia: Consejo Económico y Social de la Región de Murcia.

Saglio, Jean (1991) "Intercambio social e identidad colectiva en los sistemas industriales". Sociología del trabajo, 1, pp. 45-62.

Téllez Infantes, Anastasia (2002) "Trabajo, identidad y género: la puesta en juego de las representaciones ideológicas”. Cuadernos Relaciones Laborales 20 (1), pp. 191-214.

Wacquant, Loïc (2004) Entre las cuerdas. Cuadernos de un aprendiz de boxeador. Madrid: Alianza. 